\title{
Vortex Core Deformation and Stepper-Motor Ratchet Behavior in a Superconducting Aluminum Film Containing an Array of Holes
}

\author{
J. Van de Vondel, ${ }^{1, *}$ V. N. Gladilin, ${ }^{1,2}$ A. V. Silhanek, ${ }^{1}$ W. Gillijns, ${ }^{1}$ J. Tempere, ${ }^{2}$ J. T. Devreese, ${ }^{2}$ and V. V. Moshchalkov ${ }^{1}$ \\ ${ }^{1}$ INPAC, Katholieke Universiteit Leuven, Celestijnenlaan 200D, B-3001 Leuven, Belgium \\ ${ }^{2} T Q C$, Universiteit Antwerpen, Universiteitsplein 1, B-2610 Antwerpen, Belgium
}

(Received 25 August 2010; published 29 March 2011)

\begin{abstract}
We investigated experimentally the frequency dependence of a superconducting vortex ratchet effect by means of electrical transport measurements and modeled it theoretically using the time-dependent Ginzburg-Landau formalism. We demonstrate that the high frequency vortex behavior can be described as a discrete motion of a particle in a periodic potential, i.e., the so-called stepper-motor behavior. Strikingly, in the more conventional low frequency response a transition takes place from an Abrikosov vortex rectifier to a phase slip line rectifier. This transition is characterized by a strong increase in the rectified voltage and the appearance of a pronounced hysteretic behavior.
\end{abstract}

DOI: 10.1103/PhysRevLett.106.137003

PACS numbers: 74.78.-w, 74.25.F-, 74.25.Uv

Since the early 1990s, inspired by the pioneering work of Magnasco [1], a strong theoretical effort has been devoted to modeling complex biological ratchet systems using the one-dimensional overdamped Langevin equation of motion for a single particle [2,3]. In this approximation, the possibility of a directional motion was demonstrated for a Brownian particle, affected by an external timecorrelated driving force and a spatially periodic potential, which lacks reflection symmetry. A common prediction of these models is discretization of the particle dynamics under a periodic driving force: the average velocity of the particles can only acquire discrete values corresponding to an integer number of unit cells traveled by the particles during each cycle of the drive. Soon after these theoretical predictions were published, the main concepts were successfully applied in experimental research both at microscopic and macroscopic scales [3,4]. A particularly ideal playground with a high level of flexibility allowing us to explore the influence of particle density, particle size, and to cover the whole space of parameters of the ratchet potentials is provided by vortices in a type-II superconducting film [5]. Here, the particle is a quantized flux bundle (vortex), which is induced by an external magnetic field and which has a temperature-dependent size. These vortices can be driven by an applied electrical current flowing through the superconductor whereas the periodic asymmetric potential to trap the vortices can be generated by using modern lithographic techniques $[6,7]$.

However, two important features inherent to vortex ratchets have not been properly taken into account by the current theoretical models. First, the repulsive interaction between vortices makes their dynamics more complex. Indeed, the competition between the asymmetric pinning and the vortex-vortex repulsion leads to reversals of the preferential flow direction of vortices as their density increases $[8,9]$. Second, the one-to-one mapping of a vortex to a particle breaks down at high vortex velocities where vortex displacements take place at shorter time scales than the healing time to recover the superconducting order parameter along the vortex trajectory. As the vortex velocity is progressively increased, this latter effect leads first to a dynamic reconfiguration of the vortex lattice, followed by the elongated-vortex motion which is much faster than that of the Abrikosov vortices. The driven elongated vortices are eventually transformed into phase slip lines [10,11]. This brings us to the formulation of the main unsolved problems that will be addressed in the present work: (i) how do the internal degrees of freedom of a vortex influence the rectification properties of vortex ratchets and (ii) is it possible to realize the theoretically predicted but so far experimentally not observed vortex steppermotor behavior?

Experimental details and theoretical model. - In order to address these important issues we have investigated a superconducting vortex rectifier consisting of a $33 \mathrm{~nm}$ thick Al bridge patterned with a periodic array of asymmetric pinning sites with a unit cell consisting of two square antidots of different sizes (1200 and $600 \mathrm{~nm}$ ), separated by $400 \mathrm{~nm}$ distance [see Fig. 1(a)]. The superconducting critical temperature is $T_{c}=1.445 \mathrm{~K}$. The coherence length and the penetration depth are $\xi(0) \sim$ $73 \mathrm{~nm}$ and $\lambda(0) \sim 261 \mathrm{~nm}$, respectively. When applying an ac current along the bridge ( $x$ axis), the vortices, induced by a magnetic field perpendicular to the plane of the bridge ( $z$ axis), are subjected to a driving force in the $y$ direction. The resulting motion of vortices leads to dissipation, which is probed by measuring the voltage drop along the bridge. The superconducting vortex rectifier has been investigated both experimentally in a ${ }^{4} \mathrm{He}$ cryostat and theoretically using time-dependent Ginzburg-Landau (TDGL) simulations. The previously used theoretical approaches (Langevin dynamics, Fokker-Planck equations, molecular dynamics), which are not specific for superconducting systems, deal with motion of rigid entities 


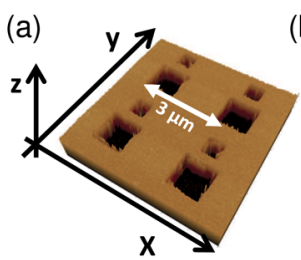

(b)
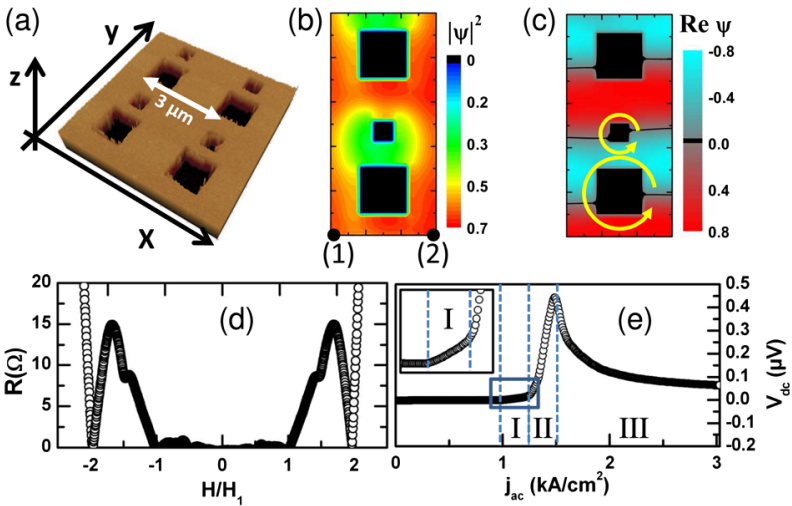

FIG. 1 (color online). (a) Atomic force microscopy image of a $5 \times 5 \mu \mathrm{m}$ area of the sample. (b) $|\psi|^{2}$ and (c) $\operatorname{Re} \psi$ calculated at $H=2 H_{1}$ using the TDGL model, with $H_{1}$ the magnetic field generating exactly one single-quantum vortex in each unit cell. Experimentally obtained (d) resistance versus field measured at $1.2 \mathrm{~K}$, and with dc current of $100 \mu \mathrm{A}$. (e) The dc voltage $\left(V_{\mathrm{dc}}\right)$, across a single row of antidots, as a function of the driving amplitude with a frequency of $100 \mathrm{kHz}$. As explained in the text, three different dynamical regimes can be identified (labeled I, II, and III) as a function of the ac amplitude. The zoom in (e) gives the voltage dependence in region I.

embedded in a medium, while vortices in a superconductor correspond to "soft" superconducting current loops which can be deformed by an applied external drive such as the Lorentz force. Unlike these approaches, the TDGL formalism $[12,13]$ treats the dynamics of the condensate as a whole, in terms of the time-dependent complex order parameter $\psi(\mathbf{r}, t)$. This treatment contains all necessary ingredients, including vortex deformability, to reproduce and explain the experimental data.

To simplify the presentation, we concentrate on the experimental data obtained at the temperature $T=1.430 \mathrm{~K}$ and in a particular magnetic field $H$, which generates one flux quantum per each antidot. The equilibrium vortex configurations are visualized by plotting the square modulus of the order parameter, $|\psi|^{2}$ [Fig. 1(b)], and its real part, $\operatorname{Re} \psi$ [Fig. 1(c)]. Since $\operatorname{Re} \psi$ should change sign 2 times along a closed path encircling a single flux quantum, Fig. 1(c) indicates the presence of one $\Phi_{0}$ vortex trapped by each antidot. The stability of this vortex configuration leads to a minimum in dissipation (i.e., resistance) at this field [14], as shown in Fig. 1(d).

Influence of the internal degrees of freedom.Figure 1(e) shows the measured rectified (dc) voltage $V_{\mathrm{dc}}$ (proportional to the average vortex velocity) as a function of the amplitude of a sinusoidal excitation at a low frequency $f=100 \mathrm{kHz}$. The positive dc voltage sign corresponds to particles moving preferentially in the $-y$ direction. This motion, opposite to the preferential direction at $H=H_{1}$, is caused by the competition between the vortex-vortex repulsion and the asymmetric pinning landscape [9]. A closer look at the amplitude dependence of $V_{\mathrm{dc}}$ reveals three distinct regions of rectification. At low ac amplitudes $j_{\mathrm{ac}}$, region I, a linear increase of $V_{\mathrm{dc}}$ is present (a close-up in the inset makes region I more visible). With increasing ac amplitude this develops into a much steeper, nonlinear increase in region II. The maximum rectification accounts for the onset of region III, where a sharp decrease of $V_{\mathrm{dc}}\left(j_{\mathrm{ac}}\right)$ takes place. A singleparticle model, as used in Refs. [1,2], cannot describe the change in the rectifying properties between regions I and II. As we demonstrate below, the sudden increase in the measured dc voltage results from a transformation of the moving vortices and their environment.

In order to elucidate the importance of the internal degrees of freedom of the vortices and the superconducting condensate for the dc voltage readout, we performed simulations using the generalized TDGL equations [13]. An important model parameter, influencing the internal structure of a moving vortex, is $\gamma=8 k_{B} T_{c} \tau_{\text {in }} \sqrt{u\left(1-T / T_{c}\right)} /$ $(\pi \hbar)$, with $\tau_{\text {in }}$ the inelastic collision time for electronphonon scattering and $u \approx 5.79$. At $\gamma \gg 1$ the healing time of the condensate is $\tau \sim \gamma|\psi| \tau_{\mathrm{GL}}$, where $\tau_{\mathrm{GL}}$ is the Ginzburg-Landau relaxation time [11]. In our calculations we have checked the behavior of the system for a wide range of $\gamma$ values. All other model parameters coincide with the experimental values.

The dependence of $V_{\mathrm{dc}}$ on the driving amplitude $j_{\mathrm{ac}}$ is shown in Fig. 2(a) for $f=400 \mathrm{kHz}$ and $\gamma=10$. The TDGL simulations reproduce the qualitative features of the experimental data [Fig. 1(e)], showing the same three distinct regions of rectification. Between 0.14 and $0.37 \mathrm{kA} / \mathrm{cm}^{2}$ an increase of the dc voltage is observed with a discontinuity at $0.36 \mathrm{kA} / \mathrm{cm}^{2}$. At this point the dependence of the dc voltage on $j_{\mathrm{ac}}$ changes from an approximately linear increase (region I, $0.14<j_{\text {ac }}$ $<0.36 \mathrm{kA} / \mathrm{cm}^{2}$ ) to a steeper nonlinear increase (region II, $0.36<j_{\mathrm{ac}}<0.37 \mathrm{kA} / \mathrm{cm}^{2}$ ).

In order to reveal the origin of these features in $V_{\mathrm{dc}}\left(j_{\mathrm{ac}}\right)$ we investigated the time evolution of the voltage difference across a single row of antidots [between points (1) and (2) in Fig. 1(b)]. At $0.33 \mathrm{kA} / \mathrm{cm}^{2}$ (region I) a clearly asymmetric motion of individual vortices is observed. A multitude of peaks in the voltage [Fig. 2(b) (I)] during one ac cycle indicates the passage of a series of vortices. A snapshot of $|\psi|^{2}$ [inset (I) of Fig. 2(a)] at maximum applied positive and negative currents indicates the presence of a vortex with a localized normal core at these low current drives. Since the dynamics of the condensate is limited by a specific time scale $\tau$, a drastic change occurs if the condensate does not have enough time to fully recover before the passage of a new vortex [11]. Under these circumstances the vortex will be deformed at higher velocities and a phase slip line (PSL) or kinematic vortex appears. A snapshot of $|\psi|^{2}$ in region II at $j_{\mathrm{ac}}=0.37 \mathrm{kA} / \mathrm{cm}^{2}$ [inset (II) of Fig. 2(a)] indeed shows the depletion of the order parameter along the direction of the vortex motion. In region II the broken symmetry of the vortex motion results 

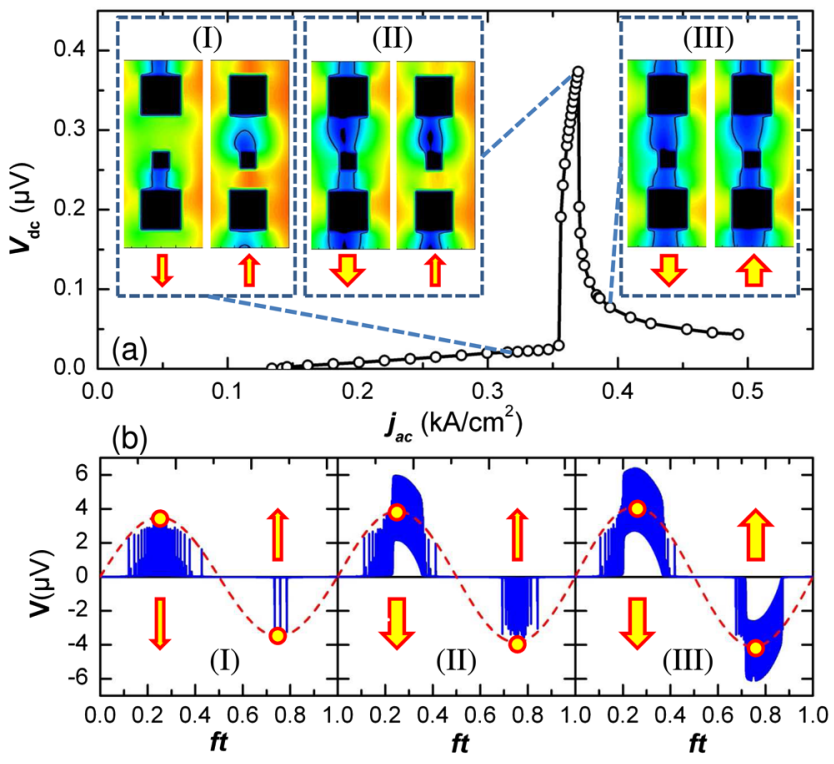

FIG. 2 (color online). (a) Calculated dc voltage $V_{\mathrm{dc}}$ as a function of the amplitude of the ac current density for $\gamma=10$ and $f=400 \mathrm{kHz}$. (b) Voltage drop between points (1) and (2) shown in Fig. 1(b), as a function of time for different ac currents: (I) $j_{\mathrm{ac}}=0.33 \mathrm{kA} / \mathrm{cm}^{2}$, (II) $j_{\mathrm{ac}}=0.37 \mathrm{kA} / \mathrm{cm}^{2}$, (III) $j_{\mathrm{ac}}=$ $0.39 \mathrm{kA} / \mathrm{cm}^{2}$. The dashed curve in (b) shows the dynamics of the applied current density. For the same ac currents, snapshots of $|\psi|^{2}$ are shown in the insets of (a), labeled, correspondingly, (I), (II), (III). The snapshots were taken during the ac cycle at maximum applied positive and negative currents. The thin and thick arrows indicate the direction of motion for Abrikosov and kinematic vortices, respectively.

in the generation of such a PSL for positive applied currents only. The transformation between the motion of vortices with a localized core to a PSL exactly coincides with the boundary between the two different regimes of rectification. The motion of kinematic vortices is much faster and results in a highly increased voltage during the positive half-period of the ac drive [Fig. 2(b) (II)] and, as such, it explains the drastic increase of $V_{\mathrm{dc}}$ in region II. Above $0.37 \mathrm{kA} / \mathrm{cm} 2$ (region III) a PSL is created during both positive and negative half-periods of the applied current [Fig. 2(b) (III) and inset (III) of Fig. 2(a)], thus giving rise to a strong reduction of the average voltage signal at higher ac drives. It is important to note that in the PSL-like regime the vortex core elongates and it is impossible to see a vortex as a single entity. In this regime, the representation of this system by a point particle model in a periodic potential breaks down. Instead, a proper treatment of the vortex within the TDGL formalism is needed to explain the observed ratchet effects.

Frequency dependence.-Since the transition from Abrikosov vortices to PSL is related to $\tau$, a change in the rectification properties is expected if the period of the driving force becomes comparable to this characteristic time scale. Unambiguous evidence of this change comes from Fig. 3 where we plotted the maximum rectified

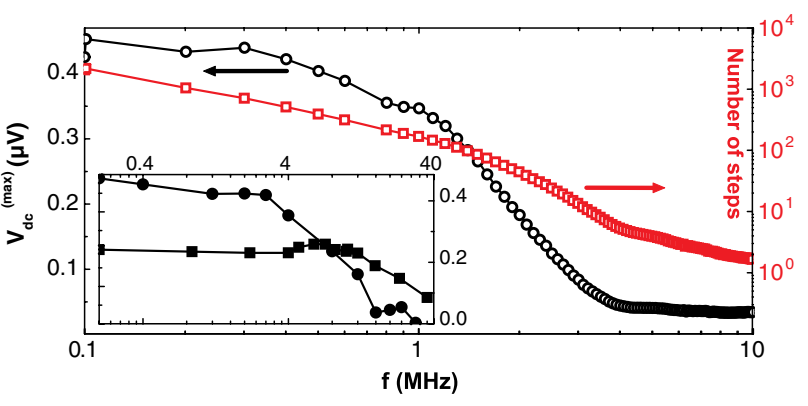

FIG. 3 (color online). Maximum rectification (open circles) and the number of steps (open squares) as a function of the frequency $f$ at $T=1.430 \mathrm{~K}$ and $H=2 H_{1}$. Inset: Maximum rectification, $V_{\mathrm{dc}}^{(\max )}(\mu \mathrm{V})$, calculated within the TDGL model for $\gamma=5$ (full squares) and 10 (full circles), as a function of $f$ $(\mathrm{MHz})$.

voltage, $V_{\mathrm{dc}}^{(\max )}$, as a function of the applied ac frequency (open circles). Above $300 \mathrm{kHz}$ a strong decrease of rectification is observed until $3 \mathrm{MHz}$, beyond which only a small decay of the rectification signal is found.

As discussed in the previous section, at low frequency drives, the maximum rectification is determined by the unidirectional creation of a PSL. The onset of PSL corresponds to the regime in which the condensate is incapable of full recovery in between two consecutive vortex passages. However, if the period of the driving force becomes comparable to the lifetime of a PSL, the PSL regime persists for both current directions. In the experiment this occurs above $3 \mathrm{MHz}$. As a result, the PSL in this regime does not significantly contribute to the rectification signal (region II disappears) and a strong reduction of the maximum rectification is expected. For those frequencies, the maximum of $V_{\mathrm{dc}}$ is achieved just before the onset of PSL, when rectification is dominated by the "conventional vortex hopping." A qualitatively similar frequency dependence is obtained in TDGL simulations [inset of Fig. 3], indicating that the model captures the main physical mechanism. A comparison between the different $\gamma$ values $[\gamma=10$ (full circles) and 5 (full squares)] shows a shift of the overall behavior towards lower frequencies at higher $\gamma$ values. This effect is explained by the healing time of the condensate $\tau$ being proportional to $\gamma$, so that the condensate acts slower for larger $\gamma$ values. In line with this, the relatively low frequency scale in the experiment can be attributed to the fact that the $\gamma$ value experimentally obtained for $\mathrm{Al}[15]$ is $\sim 500$.

Discrete rectification.-We concluded above that at sufficiently high frequencies the rectification is governed by the directional motion of Abrikosov vortices. In this regime a shorter time scale appears, associated with the traveling time of a vortex from cell to cell. The synchronization between an external excitation and the internal natural frequency should lead to a discrete hopping of vortices known as a vortex stepper motor. The average 


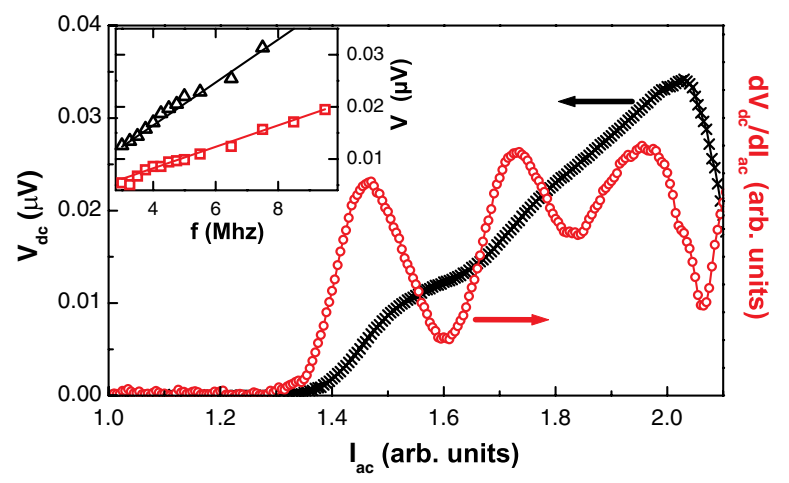

FIG. 4 (color online). $\quad V_{\mathrm{dc}}$ (crosses) and $d V_{\mathrm{dc}} / d I_{\mathrm{ac}}$ (circles) as a function of $I_{\mathrm{ac}}$ with $f=6.5 \mathrm{MHz}$. Inset: Height of the first (squares) and second (triangles) voltage step as a function of $f$. The full lines indicate the expected values $V_{\mathrm{dc}}=n_{\text {step }} \Phi_{0} f$.

voltage generated by a single moving vortex row is given by $V_{\mathrm{dc}}=n_{\text {step }} \Phi_{0} f$, with $\Phi_{0}=2.07 \times 10^{-15} \mathrm{~T} \mathrm{~m}^{2}$ the flux quantum, $f$ the frequency in $\mathrm{Hz}$, and $n_{\text {step }}$ the number of steps taken by the vortex row. Knowing $f$, we can calculate the number of the unit cells the vortex is moving during a single ac cycle (as shown in Fig. 3, open squares). At low driving frequencies, this number is large $\left(n_{\text {step }}>100\right)$. As a result, any discreteness of the vortex motion becomes difficult to resolve. However, above $3 \mathrm{MHz}$ the number of steps taken per cycle is less than 10 and discrete behavior of the vortex lattice should appear.

Figure 4 shows a representative $V_{\mathrm{dc}}\left(I_{\mathrm{ac}}\right)$ curve (crosses) in the high frequency regime $(f=6.5 \mathrm{MHz})$. Here a smoothed staircase structure is observed. The fact that the discrete hopping of vortices does not manifest itself as very sharp voltage steps can be explained by the large number of antidot rows (333) which contribute to the measured signal. This could lead to statistical spreading. A common practice to unveil these blurred steps is to measure the derivative $\frac{d V_{\mathrm{dc}}}{d I_{\mathrm{ac}}}$, as shown in Fig. 4 (circles). The minima in $\frac{d V_{\mathrm{dc}}}{d I_{\mathrm{ac}}}$ are used to determine the locations of the steps on the current axis, and the height of the steps is defined as the voltage output exactly at this location. In the inset of Fig. 4 we plotted the height of first (squares) and second (triangles) voltage step together with the theoretical prediction $V_{\mathrm{dc}}=n_{\text {step }} \Phi_{0} f$ [ gray (red) and black lines]. The nice overlap between experiment and theory demonstrates that we indeed achieved a coherent stepper-motor behavior of the whole Abrikosov lattice in the periodic pinning landscape.

To conclude, the main points of this Letter are (i) the realization of the vortex stepper motor, particularly evident at high frequency excitations, and (ii) the presence of a driving force window where the nature of the moving particle changes from an Abrikosov vortex to a phase slip line, when switching the direction of the driving force.
The former effect is very sensitive to the particular value of the magnetic field, whereas the latter is a more robust effect. The formation of phase slip lines gives rise to the experimentally observed irreversibilities which have been previously explained by attributing an artificial mass to nondeformable vortices [7]. We believe that our results have relevance for other ratchet systems of deformable objects or media [4].

This work was supported by Methusalem funding by the Flemish government, the Flemish Science Foundation (FWO-Vl), in particular, FWO projects G.0356.05, G.0115.06, and G.0370.09N, the Belgian Science Policy, and the ESF NES network. A. V.S., J. V.d. V., and W. G. acknowledge support from FWO-Vl.

*Present address: Institut Català de Nanotecnologia (ICN), Centre d'Investigació en Nanociència i Nanotecnologia (CIN2), Campus Universitat Autònoma de Barcelona, Bellaterra, Barcelona E-08913, Spain.

[1] M. O. Magnasco, Phys. Rev. Lett. 71, 1477 (1993).

[2] F. Julicher, A. Ajdari, and J. Prost, Rev. Mod. Phys. 69, 1269 (1997).

[3] P. Hänggi and F. Marchesoni, Rev. Mod. Phys. 81, 387 (2009).

[4] S. Matthias and F. Müller, Nature (London) 424, 53 (2003); G. Mahmud et al., Nature Phys. 5, 606 (2009); H. Linke et al., Phys. Rev. Lett. 96, 154502 (2006).

[5] C.-S. Lee et al., Nature (London) 400, 337 (1999); J. F. Wambaugh et al., Phys. Rev. Lett. 83, 5106 (1999).

[6] J.E. Villegas et al., Science 302, 1188 (2003);

[7] J. Van de Vondel et al., Phys. Rev. Lett. 94, 057003 (2005).

[8] I. Derényi and T. Vicsek, Phys. Rev. Lett. 75, 374 (1995); W. Gillijns et al., Phys. Rev. Lett. 99, 247002 (2007); S. Savel'ev, F. Marchesoni, and F. Nori, Phys. Rev. Lett. 91, 010601 (2003); 92, 160602 (2004); Q. Lu, C. J. Olson Reichhardt, and C. Reichhardt, Phys. Rev. B 75, 054502 (2007).

[9] C.C. de Souza Silva et al., Nature (London) 440, 651 (2006).

[10] A. I. Larkin and Yu. N. Ovchinnikov, Zh. Eksp. Teor. Fiz. 68, 1915 (1975) [Sov. Phys. JETP 41, 960 (1975)]; A. Andronov et al., Physica (Amsterdam) 213C, 193 (1993); A. G. Sivakov et al., Phys. Rev. Lett. 91, 267001 (2003); A. V. Silhanek et al., Phys. Rev. Lett. 104, 017001 (2010).

[11] D. Y. Vodolazov and F. M. Peeters, Phys. Rev. B 76, 014521 (2007).

[12] L. P. Gor'kov and G. M. Eliashberg, Zh. Eksp. Teor. Fiz. 54, 612 (1968); [Sov. Phys. JETP 27, 328 (1968)].

[13] L. Kramer and R. J. Watts-Tobin, Phys. Rev. Lett. 40, 1041 (1978).

[14] M. Baert et al., Europhys. Lett. 29, 157 (1995); E. Rosseel et al., Phys. Rev. B 53, R2983 (1996).

[15] W. E. Lawrence and A. B. Meador, Phys. Rev. B 18, 1154 (1978). 